\title{
The Kronecker Power of a Permutation*
}

\section{Russell Merris**}

(March 22, 1976)

\begin{abstract}
Let $G$ be a permutation group of degree $n$. Think of the elements, $\sigma$, of $G$ as $n$-square permutation matrices. The paper concerns a reduction of the representation $\sigma \rightarrow$ the $r$ th Kronecker power of $\sigma$. In case $G$ is the full symmetric permutation group, a formula is given which involves the Stirling numbers of the second kind.
\end{abstract}

Key words: Bell numbers; branching theorem; Clebsch-Gordon series; irreducible character; matrix functions; multiple transitivity; permutation group; Stirling numbers.

\section{Introduction}

Let $S_{n}$ denote the full symmetric permutation group of degree $n$. For each $\sigma \epsilon G$, let $Q(\sigma)$ be the corresponding permutation matrix, i.e., $Q(\sigma)=\left(\delta_{i \sigma(j)}\right)$. If $G$ is any subgroup of $S_{n}$, then $Q$ is a faithful representation of $G$ whose character, $\theta$, counts the number of fixed points. In this note, we investigate a reduction of $\theta^{r}$, the character of the $r$ th Kronecker power of $Q$.

The reduction of the Kronecker (or inner) product of two irreducible representations is called a Clebsch-Gordon series. When $G=S_{n}$, the problem of obtaining a Clebsch-Gordon series has been solved (see, e.g., [3], ${ }^{1}$ [4] or [7]). However, the solution does not easily lead to explicit formulas for the reduction of higher Kronecker powers of representations.

When $1 \leq r \leq n$, the problem naturally arises in connection with a certain class of matrix functions: Let $\lambda$ be an irreducible character of $G$. If $A=\left(a_{i j}\right)$ is an $n$-square complex matrix, let

$$
e_{r}(A)=\sum_{\sigma \in G} \lambda(\sigma) E_{r}\left(a_{1 \sigma(1)}, \ldots, a_{n \sigma(n)}\right)
$$

where $E_{r}$ is the rth elementary symmetric function. It is easily seen, by making special choices for $r$, $G$, and $\lambda$, that determinant, permanent and trace are all examples of $e_{r}$ functions. It is proved in [5, Theorem 6] that $e_{r}$ is not identically zero, if and only if $\lambda$ is a component of $\theta^{r}$. Thus, although our general interest is to obtain a reduction of $\theta^{r}$, we are specifically interested in the smallest number $k$ such that $\lambda$ is a component of $\theta^{k}$.

\section{Results}

If $\lambda$ and $\chi$ are characters of $G$, then by $(\lambda, \chi)_{G}$ we denote the usual "inner product" of characters. Let $\Gamma_{r, n}$ be the set of functions from the first $r$ to the first $n$ positive integers. It will sometimes be convenient to think of a function $\gamma \in \Gamma_{r, n}$ as a sequence, $\gamma=(\gamma(1), \gamma(2), \ldots, \gamma(r))$. The $r$ th Kronecker power of $Q(\sigma)$ is an $n^{r}$-square matrix which is indexed by $\Gamma_{r, n}$ (usually ordered lexicographically). For $\alpha, \beta \in \Gamma_{r, n}$, the $\alpha, \beta$ entry of this big matrix is $\prod_{t=1}^{r} \delta_{\alpha \alpha(t), \sigma \beta(t)}=\delta_{\alpha, \sigma \beta}$. In

AMS Subject Classification: Primary, 20C30; Secondary, 15A15, 05A19.

*An invited paper. A portion of this work was done during the period when the author was supported by a research grant from the National Science Foundation.

**Present address: Department of Mathematics, California State University, Hayward, CA 94.542.

${ }^{1}$ Figures in brackets indicate the literature references at the end of this paper. 
particular,

$$
\theta^{r}(\sigma)=\sum_{\gamma \in \Gamma_{r, n}} \delta_{\gamma, \sigma \gamma}
$$

For $\gamma \in \Gamma_{r, n}$, define $G(\gamma)=\{\sigma \epsilon G: \sigma \gamma=\gamma\}$. It is straight forward to verify that $G(\gamma)$ is the subgroup of $G$ which individually fixes the elements of the range of $\gamma$.

LEMMA: Let $\lambda$ be an irreducible character of $\mathrm{G}$. Then

$$
\left(\lambda, \theta^{\mathrm{r}}\right)_{\mathrm{G}}=\underset{\gamma \in \Gamma_{\mathrm{r}, \mathrm{n}}}{\sum_{\mathrm{f}}}[\mathrm{G}: \mathrm{G}(\gamma)]^{-1}(\lambda, 1)_{\mathrm{G}(\gamma)} .
$$

Proof:

$$
\begin{aligned}
\left(\lambda, \theta^{r}\right)_{G} & =\frac{1}{o(G)} \sum_{\sigma \in G} \lambda(\alpha) \theta^{r}(\sigma) \\
& =\frac{1}{o(G)} \sum_{\sigma \in G} \sum_{\gamma \in \Gamma_{r, n}} \lambda(\sigma) \delta_{\gamma, \sigma \lambda} \\
& =\frac{1}{o(G)} \sum_{\gamma \in \Gamma_{r, n}} \sum_{\sigma \in G(\gamma)} \lambda(\sigma) \\
& =\sum_{\gamma \in \Gamma_{r, n}}[G: \quad G(\gamma)]^{-1}(\lambda, 1)_{G(\gamma)} .
\end{aligned}
$$

Let $Q_{r, n}$ be the subset of $\Gamma_{r, n}$ consisting of strictly increasing functions, i.e., $\gamma \in Q_{r, n}$ if and only if $1 \leq \gamma(1)<\gamma(2)<\ldots<\gamma(r) \leq n$. Since $G(\gamma)$ consists of those $\sigma \in \mathrm{G}$ which individually fix the integers in the range of $\gamma$, it follows that $G(\gamma)=\{i d\}$ for all $\gamma \in Q_{n-1, n}$. In particular, $(\lambda, 1)_{G(\gamma)}=$ $\lambda(i d)$ for all $\gamma \in Q_{n-1, n}$. It follows from the lemma that $\left(\lambda, \theta^{n-1}\right)_{\mathrm{G}}>0$ for every irreducible character $\lambda$ of $G$. Since the principal (identically one) representation is a component of $Q$ (i.e., $\left.(1, \theta)_{G}>0\right)$ we see that $\left(\lambda, \theta^{r}\right)_{G} \leq\left(\lambda, \theta^{r+1}\right)_{G}$. These remarks are restated as

Conollary 1: Let $\mathrm{G}$ be a subgroup of $\mathrm{S}_{\mathrm{n}}$. There exists a positive integer $\mathrm{m} \leq \mathrm{n}-1$ such that ( $\chi$, $\left.\theta^{\mathrm{r}}\right)_{\mathrm{G}}>0$ for every irreducible character $\chi$ of $\mathrm{G}$ and every $\mathrm{r} \geq \mathrm{m}$.

In general, we may expect a randomly chosen irreducible character to appear in $\theta^{\mathrm{r}}$ for some $\mathrm{r}<\mathrm{m}$.

Corollary 2: Let $\lambda$ be an irreducible character of G. Suppose $\mathrm{k}$ is the smallest integer such that $\left(\lambda, \theta^{\mathbf{k}}\right)_{\mathrm{G}}>0$. Then

$$
\left(\lambda, \theta_{\cdot}^{\mathbf{k}}\right)_{\mathrm{G}}=(\mathrm{k} !) \sum_{\gamma \in Q_{\mathrm{k}, \mathrm{n}}}[\mathrm{G} ; \mathrm{G}]^{-1}(\lambda, \mathrm{l})_{\mathrm{G}(\gamma)}
$$

Proof: Take $\beta \epsilon \Gamma_{k, n}$. Suppose $\beta$ contains exactly $s$ distinct integers. Let $\alpha \in Q_{s, n}$ be the sequence which contains the distinct integers appearing in $\beta$. Then $G(\alpha)=G(\beta)$. If $s<k$, then by (1) and the definition of $k,(\lambda, 1)_{G(\alpha)}=0$. Therefore, for $r=\mathrm{k}$, the only terms of (l) which survive correspond to sequences of distinct integers. These are precisely $\left\{\gamma \sigma: \quad \gamma \in Q_{k, n}, \sigma \in S_{k}\right\}$.

In what follows, we will frequently take $G=S_{n}$. In this case, we write $(\lambda, \chi)_{n}$ rather than the more cumbersome $(\lambda, \chi)_{S n}$. 
Corollary 3: Let $\lambda$ be an irreducible character of $\mathrm{S}_{\mathrm{n}}$. For $\mathrm{l} \leq \mathrm{r}<\mathrm{n}$,

$$
\left(\lambda, \theta^{r}\right)_{n}=\sum_{t=1}^{r} S(r, t)(\lambda, 1)_{n-1},
$$

where the numbers $\mathrm{S}(\mathrm{r}, \mathrm{t})$ are Stirling numbers of the second kind.

Proof: Let $\gamma$ be a generic element of $\Gamma_{r . n}$. For each $t=1,2, \ldots, r$, there are $S(r, t)$ ways of partitioning the $r$ components of $\gamma$ into $t$ nonempty subsets. For each such partition, there are $n ! /(n$ $-t$ )! ways of filling the $r$ positions in $\gamma$ in such a way that the same integer appears in two components if and only if both components belong to the same set of the partition. In other words, there are $n ! S(r, t) /(n-t)$ ! sequences in $\Gamma_{r, n}$ which contain precisely $t$ distinct integers. Finally, if $G$ $=S_{n}$, then $G(\gamma)$ consists of the symmetric group on the $n-t$ integers not appearing in $\gamma$. The result follows from eq (1).

The numbers

$$
b_{r}=\sum_{t=1}^{r} S(r, t)
$$

are called Bell numbers after E. T. Bell. It follows immediately from (3) that for $1 \leq r<n,\left(1, \theta^{r}\right)_{n}$ $=b_{r}$. In fact, a more general result was proved in [6], namely for $1 \leq r \leq n,\left(1, \theta^{r}\right)_{G} \geq b_{r}$ with equality if and only if $G$ is $r$-fold transitive. Strangely, $\left(1, \theta^{n+1}\right)_{G} \geq b_{n+1}-1$, with equality if and only if $G=S_{n}$.

Corollary 4: Let $\lambda$ be an irreducible character of $\mathrm{S}_{\mathrm{n}}$. Let $\mathrm{k}$ be minimal so that $\left(\lambda, \theta^{\mathrm{k}}\right)_{\mathrm{n}}>0$. Then $\left(\lambda, \theta^{\mathrm{k}}\right)_{\mathrm{n}}=(\lambda, 1)_{\mathrm{n}-\mathrm{k}}$.

Proof. This is immediate either from Corollary 2 or Corollary 3.

An important feature of Corollary 3 is that we know how to compute $(\lambda, 1)_{n-t}$. The branching theorem [2, p. 126] states the following: Suppose $\lambda$ arises from the frame $\left(m_{1}, m_{2}, \ldots ., m_{p}\right), m_{1} \geq m_{2}$ $\geq \ldots \geq m_{p}$, and $m_{1}+m_{2}+\ldots+m_{\mathrm{p}}=n$. Then the restriction of $\lambda$ to $S_{n-1}$ decomposes as $\lambda=\lambda_{1}$ $+\lambda_{2}+\ldots+\lambda_{p}$, where $\lambda_{i}$ is the character of $S_{n-1}$ arising from the frame $\left(m_{1}, \ldots, m_{i-1}, m_{i}-1\right.$, $\left.m_{i+1}, \ldots, m_{p}\right)$, and $\lambda_{i}$ is understood not to appear if $m_{i-1}=m_{i}$. Since the identically one representation of $S_{n-t}$ corresponds to the frame $p=1, m_{1}=n-t$, we obtain the following apparently well-known result.

COROLLARY 5: Let $\lambda$ be the irreducible character of $\mathrm{S}_{\mathrm{n}}$ which arises from the frame $\left(\mathrm{m}_{1}, \mathrm{~m}_{2}, \ldots\right.$, $\left.\mathrm{m}_{\mathrm{p}}\right)$. Assume that $\lambda$ is not the principal character. The smallest number $\mathrm{k}$ such that $\left(\lambda, \theta^{\mathrm{k}}\right)_{\mathrm{n}}>0$ is $\mathrm{k}$ $=\mathrm{n}-\mathrm{m}_{1}$.

EXAMPLE 1: If $\lambda=\epsilon$, the alternating character of $S_{n}$, then $\lambda$ corresponds to the frame $p=n$, $m_{1}=m_{2}=\ldots=m_{n}=1$. In particular, by Corollary $5,\left(\epsilon, \theta^{r}\right)=0$ unless $r \geq n-1$. (This proves that the bound in Corollary 1 is sharp.) Using Corollary 4 , we find that $\left(\epsilon, \theta^{n-1}\right)_{n}=(\epsilon, 1)_{1}=1$.

ExAmple 2: Let $G=S_{5}$. Let $\lambda$ be the character arising from the frame $(2,2,1)$. From Corollary 5 , the smallest $k$ for which $\left(\lambda, \theta^{k}\right)_{5}>0$ is $k=5-2=3$. Using Corollary 4 and the branching theorem, we obtain $\left(\lambda, \theta^{3}\right)_{5}$ : Confusing the frame with the character, the restriction of $(2,2,1)$ to $S_{4}$ is $(2,1,1)+(2,2)$. The further restriction to $S_{3}$ is $(1,1,1)+(2,1)+(2,1)$. Finally, the restriction of $(2,2,1)$ to $S_{2}$ is $3(1,1)+2(2)$. By Corollary $4,\left(\lambda, \theta^{3}\right)_{5}=(\lambda, 1)_{2}$. But we have just discovered that $(\lambda, 1)_{2}=2$.

I am grateful to Kenneth R. Rebman for pointing out to me that the numbers in (3) are the Stirling numbers, and to the anonymous referee of an earlier effort for the brief proof of Corollary 3. My original proof, involving the Frobenius Reciprocity Theorem for induced characters, is substantially more complicated; it does not rely on the lemma. 


\section{References}

[1] Berge, C., Principles of Combinatorics (Academic Press, New York-London, 1971).

[2] Boerner, Hermann, Representations of Groups with Special Consideration for the Needs of Modern Physics (NorthHolland/American Elsevier, Amsterdam-London/New York, 1970).

[3] Coleman, A. J., Induced Representations with Applications to $S_{n}$ and $G L(n)$, Queen's Papers in Pure and Applied Math. (Queen's University, Kingston, Ontario, 1966).

[4] Hamermesh, Morton, Group Theory and its Application to Physical Problems, (Addison-Wesley, Reading-London, 1962).

[5] Merris, Russell, Inequalities for matrix functions, J. Algebra 22, 451-460 (1972).

[6] Merris, Russell, and Pierce, Stephen, The Bell numbers and $r$-fold transitivity, J. Combinatorial Theory 12, 155-157 (1972).

[7] Robinson, G., de B. Representation Theory of the Symmetric Group (Univ. of Toronto Press, 1961).

(Paper 80B2-441) 